\title{
Experimental Evaluation of Actuation and Sensing Capabilities of a Haptic Device
}

\author{
E. Mobedi, I. Görgülü and M.I.C. Dede
}

Izmir Institute of Technology, Turkey, e-mails: \{emirmobedi, ibrahimcangorgulu, candede\}@iyte.edu.tr

\begin{abstract}
.
Haptic devices are used to increase the telepresence level by providing the sense of touch to the human operator. Simultaneously, they capture the targeted motion of the human operator to generate a motion demand for the teleoperated slave system. Considering a scenario where the slave system's end-effector is handled by the human operator at the master side, which is attached to the haptic device, an ideal haptic interaction involves the feeling of only the end-effector dynamics and the accurate sensation of the end-effector pose. The performance of a haptic device is based on these two functionalities. In this paper, the experimental evaluation of the actuation and sensing capabilities of a haptic device, HIPHAD v1.0 kinesthetic haptic device, is presented.
\end{abstract}

Key words: Haptic device performance, Experimental performance evaluation, System identification

\section{Introduction}

Haptic devices are used for a variety of tasks such as surgical robotics [11], robotic rehabilitation [3]. These devices are categorized into two groups in terms of feedback types as kinesthetic and tactile. Kinesthetic haptic devices are developed to stimulate bodily position, weight, or movement of the muscles, tendons, and joints [9] while measuring the pose of the human's targeted motion. Therefore, a kinesthetic haptic device is used both as a sensory and an actuation system.

Back-drivability is defined in the literature as the level of easiness of the transmission of movement from the end-effector to the input axes [6]. In this definition, user handles the end-effector and the actuators are coupled with the input axes. If back-drivability can be achieved without any power input to the device, this type of a haptic device is called impedance-type haptic device [7]. In an ideal case, if the haptic device is intended to act as a lumped mass of the tool, the force felt by the user should be proportional to the acceleration of this lumped mass. In the case of a 
physical interaction, interaction forces are calculated based on the impact velocity, mass, damping and stiffness of the objects and slave system [2].

Mass, damping and stiffness properties of a haptic device manipulator are defined as the impedance of the device. If the inertial, damping and stiffness properties of the device are identified with some accuracy, a feedforward term can be utilized in order to cancel the effect of these dynamics [11]. Hence, when there is no contact, the user feels almost only the resistance due to the tool inertia and this situation increases the back-drivability performance of the system or in other words, minimizes the minimum impedance. The maximum impedance that a haptic device can display is related to its actuation capacity which depends on the inertial, damping and stiffness properties of the device and actuation system located at the input axes. Therefore, actuation performance evaluation of a haptic device is important in order to characterize the system and also to enlarge the impedance range by control.

The measured pose of the targeted human motion by the haptic device is used to transmit motion demand to a slave system. In precise operation the resolution and the repeatability of this sensory acquisition system become important.

A number of methods have been proposed for evaluation of actuation and sensing capabilities of haptic devices [4], [10], [11]. The aim of this study is to evaluate actuation and sensing capabilities of a haptic device, HIPHAD v1.0, by determining its impedance parameters, and workspace position resolution and repeatability.

\section{Kinematics and Static Force Analysis of HIPHAD}

In this section, the results of the kinematics and static force analysis of HIPHAD v1.0 are presented. The device was designed and produced in IzTech Robotics Laboratory as an open-loop impedance-type structure with the hybrid mechanism. The translational parallel mechanism of the device has three actuated degrees-of-freedom (DoF). The mechanism is a modified version of the R-CUBE mechanism and the modifications are described in [1]. Forward kinematics equation of the mechanism is shown in Eq. (1).

$$
W_{r i}=S+l_{i 1} \cdot \sin \theta_{i} ; i=1,2,3
$$

Here, $W_{r i}$ indicates the end-effector location with respect to the origin along the $\vec{u}_{i}$ direction, $S$ parameter is the distance of the actuation axis from the origin, $\theta_{i}$ represents the joint variable, $l_{i 1}$ is the effective link length and $i$ represents the $i^{t h}$ serial chains and $\vec{g}$ is the gravity vector as indicated in Fig.1. The map of the endeffector force to the actuator inputs is shown as follows;

$$
T_{i}=F_{i} \cdot l_{i 1} \cdot \cos \theta_{i} ; i=1,2,3
$$


where $F_{i}$ represents the force applied by the device in the task space along the $\vec{u}_{i}$ direction and $T_{i}$ indicates the torque generated by the actuator connected to the $i^{\text {th }}$ serial kinematic chain. Detailed analyses of the kinematics and quasi-static force are presented in [5].
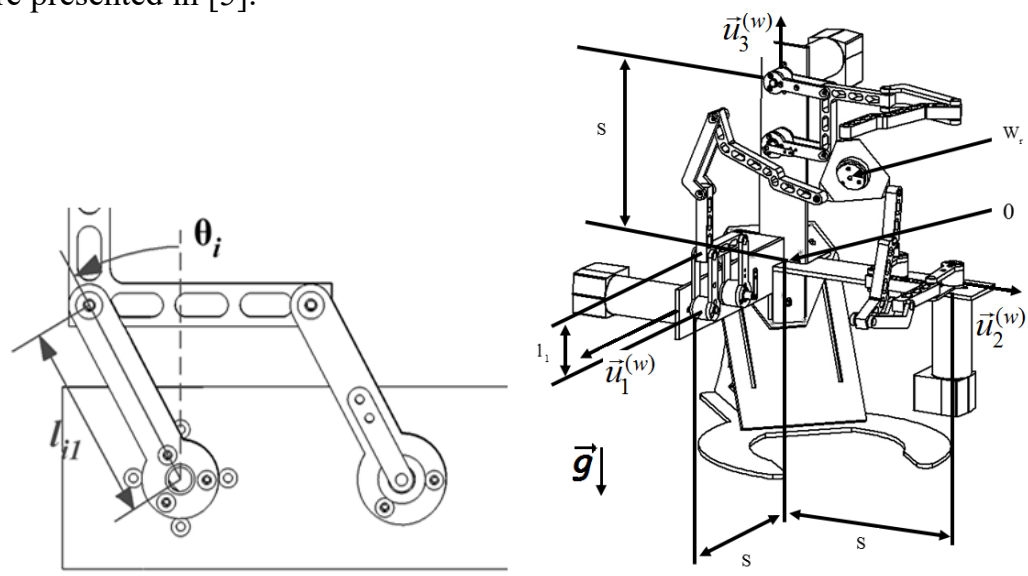

Fig. 1 Kinematic parameters of HIPHAD v1.0

\section{Actuation Capabilities}

In this section, frequency response method is explained to identify the actuation capability of HIPHAD v1.0. For an impedance-type haptic interface, a sweep force signal is applied to the actuators over a certain range of frequencies at a certain amplitude and velocity and force responses are measured [11]. Afterwards, the behavior of the system is identified by making use of the experimentally obtained Bode diagrams. Then, a transfer function is fitted to determine the model of the system. In other words, actuation performance of a haptic device is determined. During the tests, no control strategy is employed on the system. However, in our setup, in order to cancel the nonlinear effects of gravity, a feedforward term is used for gravity compensation. The test was carried out only for $\vec{u}_{1}$ axis since all axes shares the same structure and the actuation of the system is uncoupled along the three axes. The steps of the experiment are presented as follows:

$\mathbf{1}^{\text {st }}$ step: A gravity compensation algorithm is employed as explained in [5].

$2^{\text {nd }}$ step: The input axis' joint position is set to $\theta_{1}=0^{\circ}$ position before each test and the other input axes are mechanically fixed at the same angular position.

$3^{\text {rd }}$ step: An accelerometer (Type: CXL10GP3, MEMSIC Brand) is attached to the end-effector as presented in Fig.2 by (1) and sinusoidal end-effector force ranging from 0.1 to $50 \mathrm{~Hz}$ is modeled and then translated as joint torque inputs by using Eq. 2. The amplitude of the end-effector force is kept at $0.17 \mathrm{~N}$ between $0.1-0.9 \mathrm{~Hz}$ 
and $0.5 \mathrm{~N}$ for the rest. During the tests, the accelerometer data were collected after the system has reaches a steady-state condition in the operating frequency.

$4^{\text {th }}$ step: The accelerometer data is processed and then integrated to calculate the end-effector velocity. By calculating the ratio between the output velocity and input force, modified admittance Bode diagram $\left(V_{\text {out }} / F_{\text {in }}\right)$ is obtained as shown in Fig. 3. This technique is defined in the literature as the open-end experiment [11].

$5^{\text {th }}$ step: In this step, force response of the system is evaluated. A 6-DOF force/torque sensor system (type: Mini45, calibration: SI-580-20, with a DAQ called Netbox from ATI Industrial Automation, Inc.) indicated as (2) in Fig. 2 is used to measure the output force. First, the end-effector is fixed mechanically and the force sensor is attached between the end-effector and the fixture (Fig. 2.1). Then, the similar procedure expressed in the $3^{\text {rd }}$ step is carried out. However, the force amplitude for the whole frequency range is set to $3 \mathrm{~N}$ and the output forces are acquired through the force sensor. Finally, the Bode diagram of force response is plotted for $F_{\text {out }} / F_{\text {in }}$ as illustrated in Fig. 3. This method is indicated in the literature as the fixed-end experiment [11].

$6^{\text {th }}$ step: A final calculation is made to find the ratio of the force response of the fixed-end frequency experiment to the velocity response of the open-end experiment. Thus, Bode diagram for $F_{\text {out }} / V_{\text {out }}$ of the device is obtained.

$7^{\text {th }}$ step: Finally, a second order mass-spring-damper in parallel connection model is fitted to the impedance Bode diagram $\left(F_{\text {out }} / V_{\text {out }}\right)$ as shown in Fig. 3.

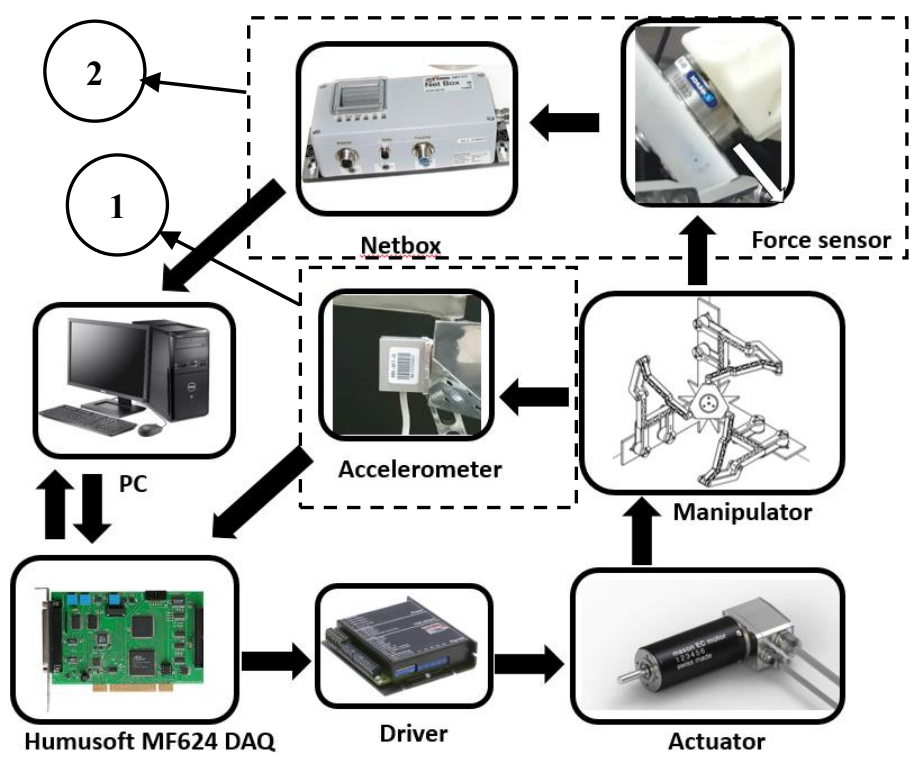

Fig. 2 Information flow of the experimental setups for frequency response method:

(1) Fixed-end experiment (2) Open-end experiment 
According to Fig.3; fixed-end experiment results revealed that the applicable force range (bandwidth) is up to $10 \mathrm{~Hz}$ and a natural frequency is observed at $30 \mathrm{~Hz}$ which is caused due to the dynamics of the actuator (Type: EC 45, Maxon motor).

$$
Z_{\text {device }}=\frac{F_{\text {out }}}{V_{\text {out }}}=\frac{m s^{2}+b s+k}{s}=K_{P} \cdot \frac{s^{2}+2 \zeta w_{n} s+w_{n}^{2}}{w_{n}^{2} s}=\frac{0.63 s^{2}+3.184 s+25}{s}
$$

Investigating the impedance plot of the total system, it is clear that the corner frequency of the system is found to be around $1 \mathrm{~Hz}$. Comparing the transfer functions presented in Eq. 3, the system's equivalent mass is calculated to be $0.63 \mathrm{~kg}$, its damping is $3.184 \mathrm{Ns} / \mathrm{m}$ and stiffness is $25 \mathrm{~N} / \mathrm{m}$. The mass of the model is compared with the CAD model of the manipulator and the error is found to be $3 \%$ between the real mechanism and the modelled one.

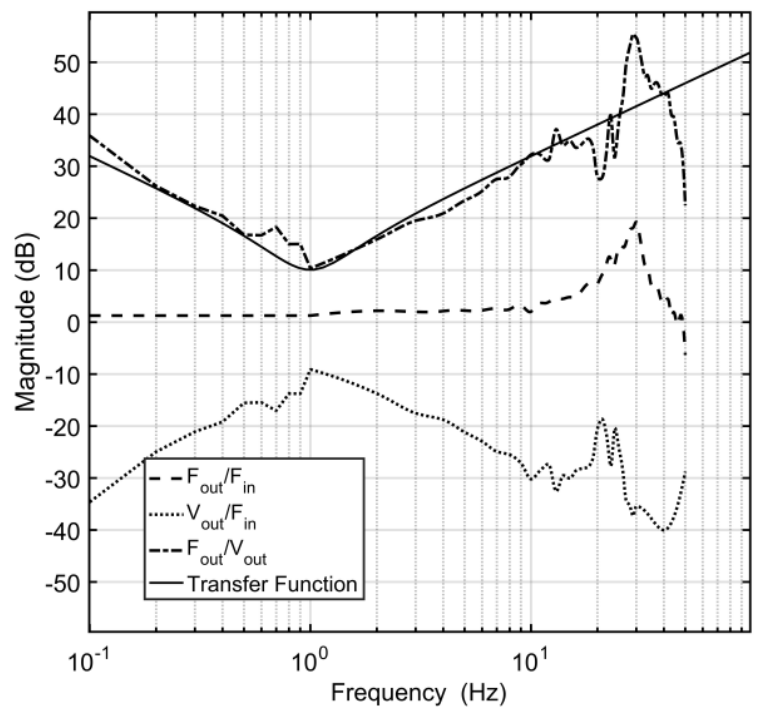

Fig. 3 Bode diagram of obtained data and fitted transfer function.

\section{Sensing Capabilities}

\subsection{Workspace Position Resolution}

In this section, the position resolution of the $\vec{u}_{1}$ axis is studied. Position resolution is indicated as a distance which is obtained through the minimum applicable encoder resolution [8]. Therefore, the experiment is conducted by following this criterion and the details of the procedure are described as follows: 
$1^{\text {st }}$ step: Theoretical workspace resolution is calculated through the forward kinematics in Eq. (1) for the encoder which has $0.087890625^{\circ}$ resolution per step.

$2^{\text {nd }}$ step: Five test points are chosen along the test axis by examining the most critical points from the theoretical workspace resolution and the end-effector is situated at these points, respectively. In addition, the dial indicator is located at these points, for external measurement of end-effector location.

$3^{\text {rd }}$ step: At the first test point, the motor is driven for two counts change in encoder by using a position controller. Afterward, the end-effector position variation is measured by the help of dial indicator, which has 0.01 resolution. The information flow of the experiment is presented in Fig. 4. The reason for the two counts change is that single step count could not be followed by the controller without overshoot. During the experiment, the dial indicator was in contact with the end-effector so that there was no gap between the end-effector and the dial indicator.

$4^{\text {th }}$ step: Finally, the differences between the measured and calculated end-effector positions are calculated and presented in Table 1.

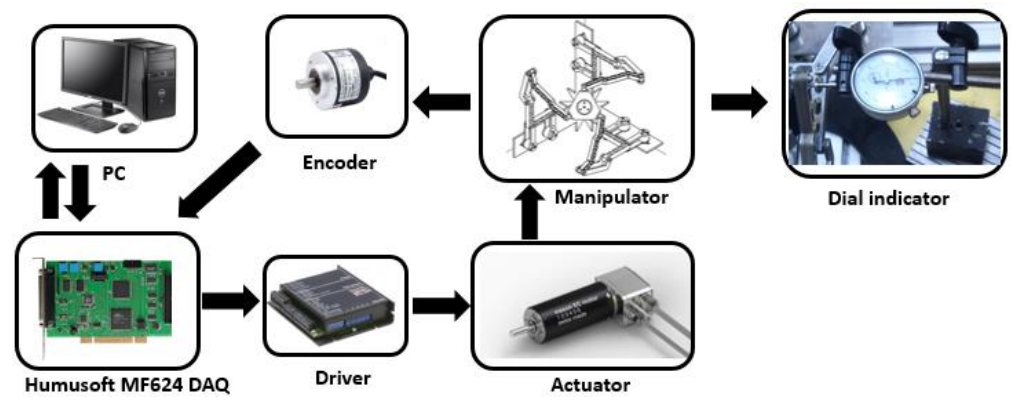

Fig.4 Information flow of the experimental setup of the position resolution test

Table 1 Results of the workspace resolution experiment

\begin{tabular}{|c|c|c|c|}
\hline Test range (degree) & Measured resolution $(\mathrm{mm})$ & Theoretical resolution $(\mathrm{mm})$ & Error value $(\mathrm{mm})$ \\
\hline 59.77 & 0.11 & 0.1 & 0.01 \\
\hline 41.20 & 0.17 & 0.15 & 0.02 \\
\hline 0.021 & 0.2 & 0.19 & 0.01 \\
\hline $\begin{array}{ll}-39.57 & -39.74\end{array}$ & 0.17 & 0.15 & 0.02 \\
\hline$-60.20-60.28$ & 0.13 & 0.1 & 0.03 \\
\hline
\end{tabular}

According to the Table 1, it is obvious that there is a relatively large difference in terms of resolution between the $0^{\circ}$ and $59.94^{\circ}$ test ranges. The reason arises from the nonlinear kinematic relationship between the joint space and task space which is indicated in Eq. 1. Therefore, the mechanism has the capacity to achieve precise operations if the links are located away from $0^{\circ}$ position. On the other hand, the errors are observed because of joint clearance as well as the compliance of the manipulator. Moreover, the spring of the dial indicator may have affected the results. 


\subsection{Workspace Position Repeatability}

In this section, position repeatability of the $\vec{u}_{1}$ axis is presented. The repeatability term is expressed as the numerical position error which occurs when the end-effector is moved to a predefined point for several times [12]. The same test setup explained in Fig. 4 is used to measure the position differences at the end-effector. Moreover, the "Statistical testing of the operational and positional accuracy of machine tools basis-VDI/DGQ 3441" is implemented as follows:

$\mathbf{1}^{\text {st }}$ step: Three test points are specified with unequal distance from each other so that recurrent errors are detected. Also, test points are chosen by evaluating the most critical points of the workspace.

$2^{\text {nd }}$ step: The positive initiation point, which is the starting point of the end-effector, is located at $+60 \mathrm{~mm}$ along the workspace for (-) direction measurement. Afterwards, the dial indicator and the end-effector are positioned at the first test point $(+1.22 \mathrm{~mm})$ so that the dial indicator is set to zero by contacting the probe of the dial indicator with the end-effector.

$3^{\text {rd }}$ step: The end-effector is moved back to the positive initiation point and then moved to the first test point. This step is repeated five times by recording the position differences that are observed at the dial indicator in Table 2.

$4^{\text {th }}$ step: The same procedure expressed in the previous step is performed for the second $(+14 \mathrm{~mm})$ and the third $(+50.2 \mathrm{~mm})$ test points.

$5^{\text {th }}$ step: For opposite direction measurement, the end-effector is located at the negative initiation point $(-10 \mathrm{~mm})$. In addition, the process described in $3^{\text {rd }}$ and $4^{\text {th }}$ step is repeated for negative direction approach.

$\mathbf{6}^{\text {th }}$ step: Finally, the standard deviation is calculated for each test point.

During the experiment, a position controller was used and the measurements were performed when the joint is moved to and stopped at the required test position.

Table 2 The repeatability test results of $\overrightarrow{\boldsymbol{u}}_{1}$ axis for both directions

\begin{tabular}{lllllll}
\hline \# of tests & $1.22 \mathrm{~mm}(-)$ & $1.22 \mathrm{~mm}(+)$ & $14 \mathrm{~mm}(-)$ & $14 \mathrm{~mm}(+)$ & $50.2 \mathrm{~mm}(-)$ & $50.2 \mathrm{~mm}(+)$ \\
\hline 1 & 0.03 & 0 & -0.02 & 0.01 & 0.07 & 0.05 \\
2 & 0.04 & 0.01 & 0 & 0 & -0.04 & 0.04 \\
3 & 0.03 & 0.01 & 0.06 & -0.08 & 0.03 & -0.03 \\
4 & 0.03 & 0.01 & 0.03 & 0.03 & 0.02 & 0.03 \\
5 & 0.02 & 0 & 0.04 & 0.02 & -0.05 & 0.04 \\
Standard & 0.006325 & 0.0054 & 0.028566 & 0.043 & 0.044989 & 0.028705 \\
Deviation & & & & & & \\
\hline
\end{tabular}

From Table 2, it is observed that the test point at $1.22 \mathrm{~mm}$ has the minimum standard deviation in both directions among the overall set of test points. Since the manipulator is operating close to its nominal position, which is also the symmetric pose, required input torque to approach this test point from both directions is almost 
identical. On the other hand, for the other test points, depending on the approach direction, the required input torque is differed more.

\section{Conclusions}

In this study, actuation and sensing capabilities of the HIPHAD v1.0 haptic device were experimentally evaluated. Making use of obtained system model, the minimum impedance of the device can be decreased by canceling the dynamic effects as required. In this way the impedance width of the device would be enlarged. In addition, the obtained experimental data will be used to guide us in developing the new version of the device.

Acknowledgments This work is supported in part by The Scientific and Technological Research Council of Turkey via grant number 117M405.

\section{References}

1. Bilgincan, T. and Gezgin, E. and Dede, M.I.C.: Integration of the Hybrid-Structure Haptic Interface HIPHAD v1.0. In: Proceedings of the International Symposium of Mechanism and Machine Theory AzCIFToMM, Izmir, Turkey (2010).

2. Colgate, J.E., and Brown J.B.: Factors Affecting the Z-Width of a Haptic Display. In: Proceedings of the IEEE International Conference on Robotics and Automation, pp. 3205-3210(1994)

3. Görgülü, I., and Maaroof O. W., and Taner B., and Dede M.I.C, and Ceccarelli M.: Experimental Verification of Quasi- Static Equilibrium Analysis of a Haptic Device. In: Proceedings of the International Symposium of Mechanism and Machine Science AzCIFToMM, Baku, Azerbaijan, pp. 57-64(2017).

4. Gupta, A. and O'Malley M.K.: Design of a Haptic Arm Exoskeleton for Training and Rehabilitation IEEE/ASME Transactions on Mechatronics, 11, pp. 280-289(2006).

5. Hayward, V., and Astley O.R.: Performance Measures for Haptic Interfaces. Robotics Research, pp. 195-206(1996).

6. Ishida, T., and Atsuo T.: A Robot Actuator Development with High Backdrivability. In: IEEE Conference on Robotics Automation and Mechatronics, pp. 1-6(2006).

7. Kern, T.A.: Engineering Haptic Devices A Beginner's Guide for Engineers. Springer(2009).

8. Martin, S., and Hillier, N.: Characterisation of the Novint Falcon Haptic Device for Application as a Robot Manipulator. In: Australasian Conference on Robotics and Automation (ACRA), pp. 1-9(2009).

9. Martinez, M. O., and Campion J., and Gholami T., Rittikaidachar M.K., Barron A.C., and Okamura A.M.: Open Source, Modular, Customizable, 3-D Printed Kinesthetic Haptic Devices. In: IEEE World Haptics Conference(WHC 2017), pp. 142-47(2017).

10. Morrell, J.B., and Salisbury J.K.: Parallel-Coupled Micro-Macro Actuators. The International Journal of Robotics Research, 17, pp. 773-791(1998).

11. Samur, E.: Performance Metrics for Haptic Interfaces. Springer(2012).

12. Taner, B.: Development and Experimental Verification of the Haptic Device, Izmir Institute of Technology(2015). 